\title{
Inmunoglobulinas en la Etiopatogenia del Liquen Plano Oral; La Evidencia Carece de Confiabilidad. Revisión Sistemática Exploratoria
}

\author{
Immunoglobulins in Etiopathogenesis of Oral Liquen Planus; \\ The Evidence Lacks Reliability. A Scoping Review
}

\author{
Belén P. Hernández ${ }^{1} \&$ Marcelo R. Sánchez²
}

\begin{abstract}
HERNÁNDEZ, B. P.\& SÁNCHEZ, M. R. Inmunoglobulinas en la etiopatogenia del liquen plano oral; la evidencia carece de confiabilidad. Revisión sistemática exploratoria. Int. J. Odontostomat., 15(3):660-665, 2021.

RESUMEN: El liquen plano oral es una enfermedad inflamatoria crónica mucocutánea, de etiología desconocida y se asocia principalmente a una respuesta de inmunidad celular. En los años 70 , se comienza a involucrar a las inmunoglobulinas en la etiopatogenia de la enfermedad y hasta la fecha la evidencia científica nos ofrece información reducida, muy variada y con resultados discutibles que hacen necesario una detallada evaluación de esta. Hemos encontrado poca investigación (32 artículos desde 1974 hasta el 2020) y la importancia relativa de las revistas donde han sido publicado los estudios es miscelánea (cuartiles 1, $2,3,4$ y no indexadas). Los artículos científicos presentan baja calidad metodológica en el 75\% (24 artículos). El 25\% restante, presenta calidad media (7 artículos) y de estos, solo el $9 \%$ (3 artículos) encontraron diferencia en la presencia de inmunoglobulinas entre los grupos de casos y controles, y un solo artículo presenta calidad metodológica alta (3\%), pero sus resultados indican que no hay información suficiente. Nuestros resultados revelan que la evidencia científica existente en cuanto a la participación de inmunoglobulinas en la etiopatogenia del liquen plano oral carece de confiabilidad por presentar calidad metodológica de baja calidad. Proponemos dejar de pensar en esta posibilidad o bien mejorar sustancialmente la metodología empleada en los futuros estudios.
\end{abstract}

PALABRAS CLAVE: liquen planus oral, immunoglobulinas, etiologia.

\section{INTRODUCCIÓN}

El liquen plano es una enfermedad inflamatoria crónica que puede afectar piel y mucosas, incluida la cavidad oral. Suele presentarse con periodos de recurrencia variable (Kurago, 2016). A menudo, involucra pacientes de mediana edad y es más común en mujeres (Mehrbani et al., 2020).

En cavidad oral puede presentarse en una amplia variedad clínica (Barbosa et al., 2014). Las lesiones suelen ser múltiples, simétricas, bilaterales e indoloras, sin embargo, las lesiones erosivas y atróficas pueden causar dolor o una sensación de ardor. Además, es considerado un desorden potencialmente malignizante, especialmente la variedad erosiva (Lu et al., 2016).
La prevalencia mundial estimada de Liquen Plano Oral (LPO) en la población adulta general es del 0,5 $\%$ al $2 \%$ (Alrashdan et al., 2016). Chile presenta una prevalencia del 1,4\% (Franch, 2017). La etiología del LPO aún se desconoce y su patogenia no está del todo aclarada. Se sabe que corresponde a una respuesta inmune celular, que inducirían una respuesta inflamatoria crónica y apoptosis de queratinocitos de la mucosa oral (Shavit et al., 2020). Esta falta de conocimiento ha desencadenado múltiples controversias y el surgimiento de nuevas teorías que han intentado explicar su etiopatogenia (Aguirre-Urizar et al., 2020). A partir de 1974, surge una serie de publicaciones científicas que involucran a las Inmunoglobulinas (Igs) en el desarrollo del LPO (Griffith et al., 1974; Lai et al., 1974). Aún

\footnotetext{
${ }^{1}$ Escuela de Odontología, Facultad de Ciencias de la Salud, Universidad de Talca, Chile.

${ }^{2}$ Escuela de Odontología, Departamento de Estomatología, Unidad de Patología y Medicina Oral, Facultad de Ciencias de la Salud, Universidad de Talca, Chile.
} 
en la actualidad, se siguen reportando investigaciones con resultados contradictorios, con muestras pequeñas, metodologías variadas, sesgos que hacen que la evidencia, hasta ahora, no tenga la calidad suficiente.

Por lo tanto, nuestro objetivo principal fue determinar la validez metodológica de la evidencia científica que relaciona la participación de las lgs en la etiopatogenia del LPO. Esto se llevó a cabo mediante la revisión completa de la literatura desde 1974 al 2020.

\section{MATERIAL Y MÉTODO}

Diseño de Estudio. Realizamos un estudio descriptivo de la literatura, tipo Revisión Sistemática Exploratoria (ScopingReview) en base al protocolo PRISMA Scr (Tricco et al., 2018).

Base de datos. Se usaron las bases de datos Pubmed, Web of Science, Scopus y Google Academic.

Búsqueda electrónica. Efectuamos una búsqueda avanzada de la literatura que incluyó artículos publicados desde enero de 1974 hasta julio del 2020. Nuestra estrategia de búsqueda para la investigación fue: (("Lichen Planus, Oral") AND "Immunoglobulins") NOT "Skin". Para otras fuentes se usó una búsqueda manual utilizando el siguiente algoritmo de búsqueda (("Lichen Planus, Oral") AND "Immunoglobulins") AND "Fluorescent Antibody Technique, Direct.

Criterios de Inclusión. Artículos científicos con fecha de publicación entre 1974-2020, sin restricción de idiomas, artículos primarios de tipo observacional y en humanos.

Criterios de Exclusión. Estudios en piel, estudios que no detallen la medición de Igs.

Selección y clasificación de los artículos. La selección de artículos científicos la realizamos en base al diagrama de flujo PRISMA Scr (Tricco et al.) con el fin de sistematizarla y evitar sesgo de selección. La revisión la realizó un investigador que seleccionó los artículos científicos, previa calibración con el segundo investigador. Esta selección se hizo según título, resumen y texto completo. Frente a diferencias, se llegó a acuerdo con el segundo investigador.

Análisis de validez interna y externa para cada artículo seleccionado. Se utilizó el "Instrumento para la lectura crítica y la evaluación de estudios epidemiológicos transversales" (Berra et al., 2008). Corresponde a un instrumento validado para evaluación de validez interna y externa de cada artículo seleccionado. Para lograr la categorización final, obtuvimos el número y porcentajes de artículos con calidad alta, media y baja.

\section{RESULTADOS}

Resultados de la búsqueda. Obtuvimos un total de 567 artículos, eliminamos 371 artículos duplicados y excluimos 141 por no cumplir con nuestros criterios de elegibilidad, en la lectura a texto completo se eliminaron 23 (por no explicitar las concentraciones de Igs y estudios de liquen plano en piel junto con mucosa oral), finalmente obtuvimos 32 artículos incluidos (Fig. 1).

Análisis bibliométrico. Entre los años 1974 - 1989 se publicaron 11 artículos (34\%), entre 1990 - 2005 hubo 4 artículos (13\%) y entre 2006 - 2020 se publicaron 17 artículos (53\%). Los países que principalmente publicaron fueron EE. UU y Dinamarca. La calidad de las revistas donde fueron publicación los artículos es miscelánea, es decir, 15 artículos (47\%) fueron publicados en revistas Q1, 5 artículos (16\%) en revistas Q2, 6 artículos (19\%) en Q3, 2 artículos (6 $\%$ ) en revistas Q4 y 4 artículos (12\%) en revistas no indexadas.

Análisis del objetivo de los artículos científicos. Los estudios tenían como objetivo determinar la presencia o concentración de Igs, o bien, otro propósito, aún así, medían concentraciones de lgs en pacientes con LPO y pacientes sanos, por lo tanto, se agruparon en función del objetivo (Tabla I).

Calidad metodológica en base a instrumento validado. Luego de analizar los artículos encontramos que, 24 artículos (75\%) poseen calidad baja y, por ende, alta probabilidad de sesgos dentro de los dominios evaluados. Solo 8 artículos poseen calidad media y alta $(25 \%)$.

Análisis de resultados de los artículos de calidad metodológica media y alta. A continuación, se analizan los resultados obtenidos en los artículos de calidad media y alta. Los artículos de calidad baja no se analizaron debido a que su calidad metodológica manifiesta alta probabilidad de sesgos, resultados cuestionables y carentes de confiabilidad. 


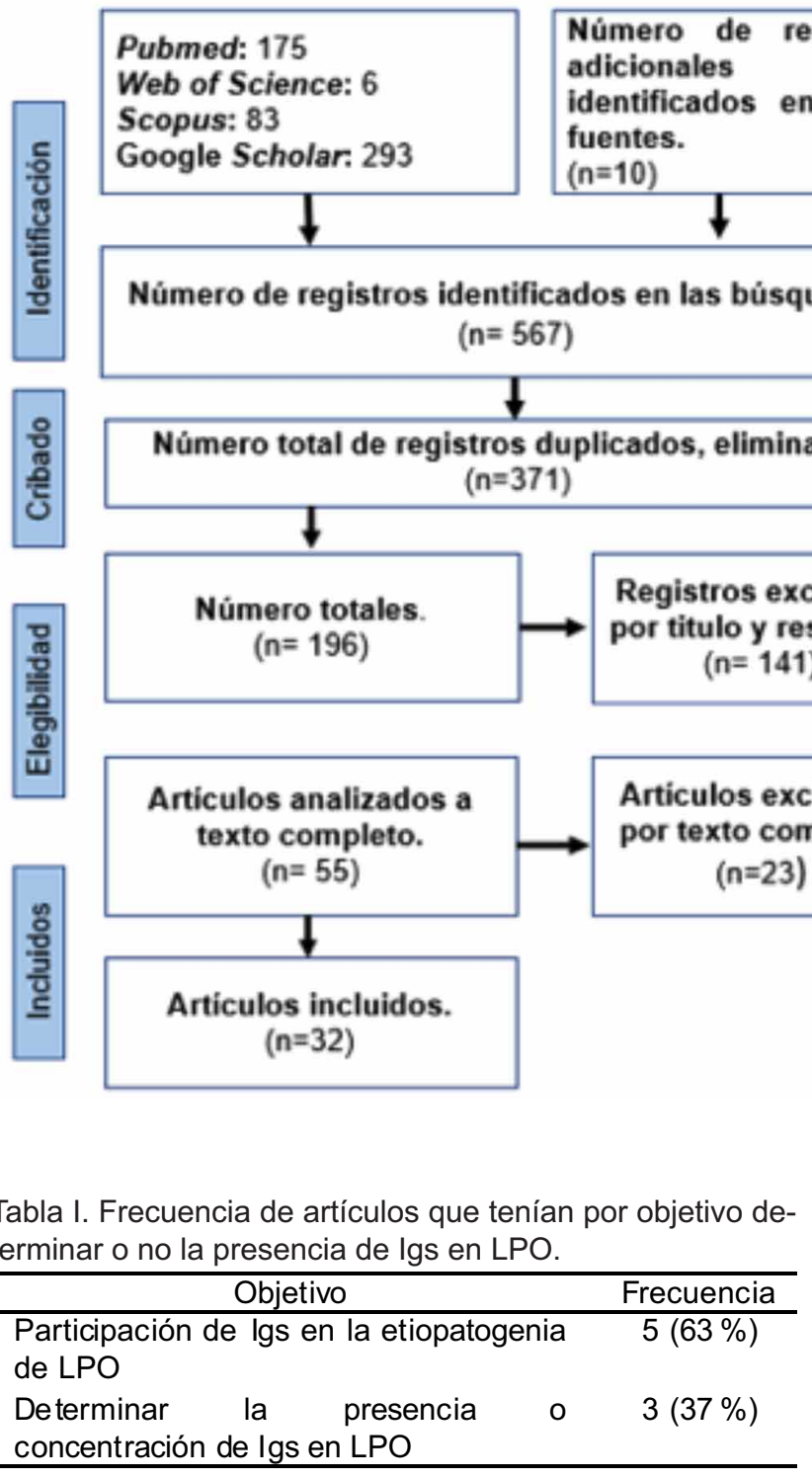

Del análisis de estos 8 artículos podemos decir que, desde el punto de vista de la calidad metodológica, 7 artículos (22\%) poseen calidad media, de los cuales, 4 artículos (13\%) no apoyan la teoría en sus conclusiones y solo 3 artículos ( $9 \%$ ) si la apoyan, pero tienen valores estadísticos imprecisos. El único artículo de calidad alta (3\%) indica que no hay información suficiente.

Según el objetivo de su investigación, 5 artículos pretendían determinar la presencia de Igs en LPO y 3 artículos, aún cuando, determinaban la presencia de Igs tenían un objetivo distinto (Tabla II).

Concluimos que el $91 \%$ de los artículos (75\% de baja calidad, $13 \%$ de media calidad y $3 \%$ de alta
Fig. 1. Diagrama de Flujo PRISMA Scr. Para la selección de artículos de forma sistematizada y libre de sesgos. calidad), no apoyan la teoría o su calidad metodológica carece de confiabilidad. Por otro lado, solo el $9 \%$ de los artículos seleccionados (de calidad media) apoya la teoría al encontrar diferencias estadísticas significativas entre los pacientes con LPO y pacientes sanos, pero de igual forma presentan observaciones metodológicas (Fig. 2).

\section{DISCUSIÓN}

Se ha creado, en la literatura científica, una base de datos que relaciona las Igs con la etiopatogenia del LPO, basada en la recopilación de antecedentes que no han sido suficientemente analizados, manteniéndose en discusión desde 1974 a la fecha. En esta revisión sistemática exploratoria denotamos una carencia de confiabilidad en los estudios.

En el análisis básico debemos mencionar la ausencia de un consenso de los niveles normales Igs en saliva y suero, lo cual impide realizar una comparación más objetiva entre los estudios realizados, ade- 
Tabla II. Análisis de Artículos de calidad media y alta.

\begin{tabular}{|c|c|c|c|}
\hline $\begin{array}{l}\text { Referencia Calidad } \\
\text { y Objetivo }\end{array}$ & $\begin{array}{c}\text { Conclusiones del artículo sobre } \\
\text { diferencia de Igs en sanos y controles }\end{array}$ & $\begin{array}{l}\text { Asociación de } \\
\text { Igs en LPO* }\end{array}$ & Observación \\
\hline $\begin{array}{l}\text { (43) } \\
\text { Media } \\
\text { No }\end{array}$ & 2 casos de 30 paciente $=6 \%$ de paciente & No & - \\
\hline $\begin{array}{l}(29) \\
\text { Alta } \\
\text { No }\end{array}$ & $\begin{array}{l}\text { No hay resultados para probar que las } \\
\text { lgs participan en el LPO. }\end{array}$ & No & - \\
\hline $\begin{array}{l}\text { (35) } \\
\text { Media } \\
\text { Si } \\
(23) \\
\text { Media } \\
\text { No } \\
\text { (9) }\end{array}$ & $\begin{array}{l}\text { Concluyen que no hay evidencia que } \\
\text { sustente una participación directa de la } \\
\text { inmunidad humoral (Ig) } \\
\text { No hay diferencia estadística entre casos } \\
\text { y controles } \\
\text { Asociación insignificante, valor } p=0.36\end{array}$ & $\begin{array}{l}\text { No } \\
\text { No }\end{array}$ & - \\
\hline $\begin{array}{l}\text { Media } \\
\text { Si } \\
(25) \\
\text { Media } \\
\text { Si }\end{array}$ & $\begin{array}{l}\text { "Los pacientes con OLP presentaron } \\
\text { peores perfiles psicológicos y trastornos } \\
\text { del sueño, así como valores más altos } \\
\text { para lgA, cortisol y proteínas totales que } \\
\text { los sujetos de control". }\end{array}$ & $\mathrm{Si}$ & $\begin{array}{l}\text { Valores de dispersión amplios. } \\
\text { Diferencias estadísticas en base a promedios. } \\
\text { Tamaño muestral de } 32\end{array}$ \\
\hline $\begin{array}{l}(14) \\
\text { Media } \\
\text { No }\end{array}$ & $\begin{array}{l}\text { "No hubo diferencias significativas en el } \\
\text { número y distribución de células IgG } \\
\text { entre LPO y otras lesiones liquenoides". }\end{array}$ & $\mathrm{Si}$ & $\begin{array}{l}\text { Tamaño muestral } 30 \text {. } \\
\text { Demuestra la presencia de IgG en el LPO y que } \\
\text { su distribución es similar a lo que presentan } \\
\text { otras lesiones liquenoides, pero no se realiza la } \\
\text { comparación con una mucosa sana, por lo } \\
\text { tanto, no se sabe si los valores son } \\
\text { significativos. }\end{array}$ \\
\hline $\begin{array}{l}(6) \\
\text { Media } \\
\text { Si }\end{array}$ & $\begin{array}{l}\text { "Los niveles elevados de } \lg \text { e e } \lg M \text { en } \\
\text { suero muestran que la inmunidad } \\
\text { humoral está implicada en la } \\
\text { patogénesis". }\end{array}$ & Si & $\begin{array}{l}\text { Los valores de IgG fueron similares. En cuanto } \\
\text { a la IgA e lgM, presentan diferencias } \\
\text { estadísticas, pero on una dispersión muy } \\
\text { amplia en los valores de los pacientes con LPO }\end{array}$ \\
\hline
\end{tabular}

*Los colores de la columna significan que, rojo no apoya la teoría y naranjo si la apoya, pero poseen observaciones, Nótese que no hay artículos de color verde, lo que apoyaría la teoría (participación de Igs en la etiopatogenia) sin objeción.

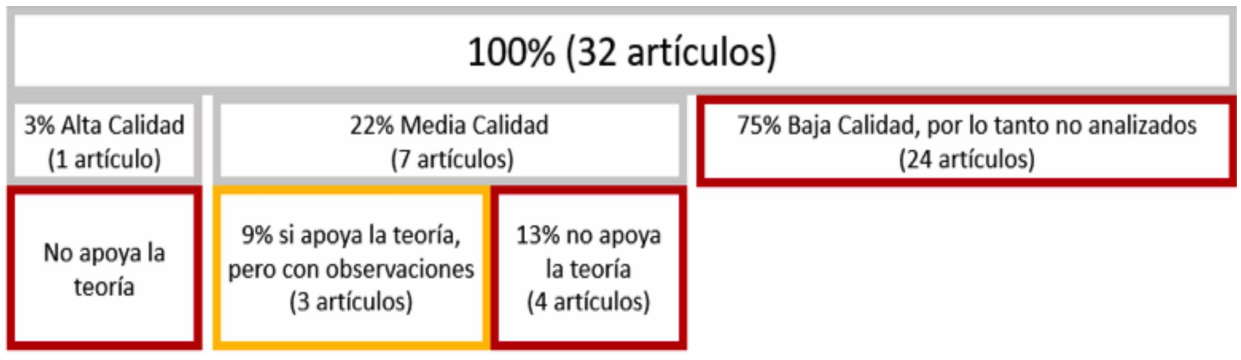

Fig. 2. Esquema resumen de acuerdo con el apoyo de la teoría de la participación de Igs en la etiopatogenia del LPO, de acuerdo con la calidad metodológica. 
más hay varios factores que influyen en las concentraciones de Igs (sexo, edad, estado físico, estado clínico de la propia enfermedad) que de no considerarlos se alterarían los resultados.

No existe en la metodología de los estudios una clara diferenciación del LPO con las lesiones liquenoides orales que presentan características clínicas e histológicas muy semejantes, pero con una etiopatogenia muy diversa, lo cual podría entorpecer o llevar a confusiones en los resultados.

Por otro lado, se presenta en la literatura una amplia gama de técnicas utilizadas para el análisis de las muestras, presentando distintos valores de especificidad y sensibilidad, además de no existir una estandarización de las mediciones semicuantitativas, lo cual, claramente podría generar distorsión en la comparación de resultados.

Finalmente, y tal vez más importante que lo anterior, es la mala calidad metodológica presente en los artículos seleccionados, medidos con el instrumento para la lectura crítica y la evaluación de estudios epidemiológicos transversales (Berra et al.).

El uso de test estadísticos en base a promedios y medianas, tienden a sesgar el valor $p$ cuando las medidas de dispersión son muy amplias, lo cual ocurre con mucha frecuencia en los artículos seleccionados. Además, una tónica repetitiva en estos estudios fue el tamaño muestral reducido que resta valor al extrapolar los resultados a una población.

La mala calidad metodológica que alcanza el $75 \%$ del total de artículos no nos permite utilizar sus resultados con confiabilidad, por lo tanto, no deberían ser usados para apoyar la teoría de la participación de lgs en el LPO. Existe un 25\% de artículos que presentan una mediana y alta calidad metodológica que, sí nos permite analizar sus resultados de manera confiable, sin embargo, solo el $9 \%$ (3 artículos) dice apoyar la teoría, aunque de igual manera, presenta variadas observaciones metodológicas. Finalmente, no encontramos artículos de buena calidad, que apoyen la teoría, presenten buenos resultados y que no sean merecedores de observaciones.

Concluimos que, la evidencia científica que relaciona las inmunoglobulinas en la etiopatogenia del liquen plano oral carece de confiabilidad. Debido a una pobre calidad metodológica, escasez de resultados positivos y presencia de observaciones.
HERNÁNDEZ, B. P. \& SÁNCHEZ, M. R. Immunoglobulins in etiopathogenesis of oral liquen planus; The evidence lacks reliability. A scoping review. Int. J. Odontostomat., 15(3):660665, 2021.

ABSTRACT: Oral lichen planus is a chronic mucocutaneous inflammatory disease of unknown etiology and is mainly associated with a cellular immune response. In the 1970s, immunoglobulins began to be involved in the etiopathogenesis of the disease and to date the scientific evidence offers us limited and highly varied information with controversial results that require a detailed evaluation of it. We found scarce research (32 articles from 1974 to 2020) and the relative importance of the journals where the studies have been published is miscellaneous (quartile 1, 2, 3, 4 and not indexed). Scientific articles present low methodological quality in $75 \%$ (24 articles). The remaining $25 \%$ presented medium quality ( 7 articles) and of these, only $9 \%$ (3 articles) found a difference in the presence of immunoglobulins between the groups of cases and controls, and only one article presented high methodological quality $(3 \%)$, but their results indicate that there is insufficient information. Our results reveal that the existing scientific evidence regarding the participation of immunoglobulins in the etiopathogenesis of oral lichen planus lacks reliability due to its low-quality methodological quality. We propose to stop thinking about this possibility or to substantially improve the methodology used in future studies.

KEY WORDS: lichenplanus, oral,
immunoglobulins, etiology.

\section{REFERENCIAS BIBLIOGRÁFICAS}

Aguirre-Urizar, J. M.; Alberdi-Navarro, J.; Lafuente-lbáñez de Mendoza, I.; Marichalar-Mendia, X.; Martínez-Revilla, B.; ParraPérez, C.; Juan-Galíndez, A. D. \& Echebarria-Goicouria, M. Á. Clinicopathological and prognostic characterization of oral lichenoid disease and its main subtypes: A series of 384 cases. Med. Oral Patol. Oral Cir. Bucal., 25(4):e554-62, 2020.

Alrashdan, M. S.; Cirillo, N \& Mccullough, M. Oral lichen planus: a literature review and update. Arch. Dermatol. Res., 308(8):53951, 2016.

Barbosa, N. G.; Silveira, É. J.; Lima, E. N.; Oliveira, P. T.; Soares, M. S. \& de Medeiros, A. M. Factors associated with clinical characteristics and symptoms in a case series of oral lichen planus. Int. J. Dermatol., 54(1):e1-e6,2014.

Berra, S.; Elorza-Ricart, J.; Estrada, M. \& Sánchez, E. Instrumento para la lectura crítica y la evaluación de estudios epidemiológicos transversales. Gac Sanit., 22(5):492-7, 2008.

Lai A Fat, R. F.; Cormane, R. H. \& van Furth, R. An immunohistopathological study on the synthesis of immunoglobulins and complement in normal and pathological skin and the adjacent mucous membranes. Br. J. Dermatol., 90(2):123-36,1974.

Franch, S. C. A. Prevalencia de lesiones de mucosa oral en adultos de 35-44 y 65-74 años en Chile. (Tesis pregrado). Facultad de Odontología, Universidad de Chile, Santiago, Chile, 2017. Disponible en: http://repositorio.uchile.cl/handle/2250/144071. 
HERNÁNDEZ, B. P.\& SÁNCHEZ, M. R. Inmunoglobulinas en la etiopatogenia del liquen plano oral; la evidencia carece de confiabilidad. Revisión sistemática exploratoria. Int. J. Odontostomat., 15(3):660-665, 2021.

Griffith, M.; Kaufman, H. S. \& Silverman, S. Studies on oral lichen planus: I. Serum immunoglobulins and complement. J. Dent. Res., 53(3):623-6,1974.

Kurago, Z. B. Etiology and pathogenesis of oral lichen planus: an overview. Oral Surg. Oral Med. Oral Pathol. Oral Radiol., 122(1):72 80, 2016.

Mehrbani, S. P.; Motahari, P.; Azar, F. P. \& Ahari, M. A. Role of interleukin-4 in pathogenesis of oral lichen planus: A systematic review. Med. Oral Patol. Oral Cir. Bucal., 25(3):e410-5, 2020.

Shavit, E.; Hagen, K. \& Shear, N. Oral lichen planus: a novel staging and algorithmic approach and all that is essential to know. F1000Res. 9:F1000 Faculty Rev-206, 2020.

Lu, S. L.; Qi, X. M.; Dong, G.; Chen, S. L.; Guo, D. W.; Wang, Y. L. \& Yang, P. S. Clinical characteristics and analysis of familial oral lichen planus in eight Chinese families. Exp. Ther. Med., 12(4):2281-4, 2016.

Tricco, A. C.; Lillie, E.; Zarin, W.; O'Brien, K. K.; Colquhoun, H.; Levac, D.; Moher, D.; Peters, M. D. J.; Horsley, T.; Weeks, L.; Hempel, S.; Akl, E. A.; Chang, C.; McGowan, J.; Stewart, L.; Hartling, L.; Aldcroft, A.; Wilson, M. G.; Garritty, C.; Lewin, S.; Godfrey, C. M.; Macdonald, M. T.; Langlois, E. V.; Soares-Weiser, K.; Moriarty, J.; Clifford, T.; Tunçalp, Ö. \& Straus, S. E. PRISMA Extension for scoping reviews (PRISMA-ScR): Checklist and explanation. Ann. Intern. Med., 169(7):467-73, 2018.

\author{
Dirección para correspodencia: \\ Belén Hernández Muñoz. \\ Odontología Universidad Talca \\ Talca \\ CHILE
}

E-mail: b.p.hernandez26@gmail.com 